[29] E. I. Jury, "Modified stability table for 2-D digital filters," IEEE Trans. Circuits Syst., vol. 35, pp. 116-119, Jan. 1988.

[30] __ "Stability of multidimensional systems and related problems," in Multidimensional Systems, Techniques and Applications, S. G. Tzafestas, Ed. New York: Marcel Dekker, March 1986, ch. 3.

[31] E. I. Jury, "Theory and applications of the Inners," Proc. IEEE, vol. 63, pp. 1044-1069, July 1975.

[32] E. I. Jury and M. Mansour, "A note on new Inner-matrix for stability," Proc. IEEE, vol. 69, pp. 1579-1580, Dec. 1981.

\section{Two-Dimensional Recursive Parameter Identification for Adaptive Kalman Filtering}

Mahmood R. Azimi-Sadjadi and Sami Bannour

\begin{abstract}
This paper is concerned with the development of a 2-D adaptive Kalman filtering by recursive adjustment of the parameters of an autoregressive (AR) image model with nonsymmetric half-plane (NSHP) region of support. The image and degradation models are formulated in a 2-D state-space model, for which the relevant 2-D Kalman filtering equations are given. The recursive parameter identification is achieved using the extension of the stochastic Newton approach to the 2-D case. This process can be implemented on-line to estimate the image model parameters based upon the local statistics in every processing window. Simulation results for removing an additive noise from a degraded image are also presented.
\end{abstract}

\section{INTRODUCTION}

The problem of adaptive Kalman filtering of nonhomogeneous images has attracted considerable attention during the recent years [1]-[6]. Space-invariant Kalman filters assume wide sense stationarity of the image field, which is not a satisfactory assumption for real world images. As a result, they are insensitive to abrupt changes and tend to smear the edges and reduce the contrast, resulting in an image with poor visual appearance. Kalman filters that use space-varying image models take into account the local statistical information within a processing window to adjust the filter parameters. One such method was introduced by Kaufman, Woods, and Tekalp [1], [2], which proposes an identification and estimation procedure for nonsymmetric half-plane (NSHP) image model that can be used on-line to evaluate the covariance matrix of the plant noise and the parameters of the AR model at each stage of the algorithm. Another approach proposed by Rajala et al. [3] is based upon partitioning an image into disjoint regions according to the local spatial activities determined by the directional derivative information. This method uses the nearest neighbor algorithm to determine the best previous state and 2-D interpolation scheme to improve the estimates of the initial states in each region. In a recent paper by Tekalp, Kaufman, and Woods [4], an edgeadaptive Kalman filter is derived that uses multiple image models to reduce the ringing artifacts that are caused by spaceinvariant filters. The selection of the appropriate model is done using the maximum a posteriori (MAP) method. Azimi-Sadjadi [5], [6] introduced a 2-D adaptive block Kalman filtering, which is used to remove the effects of speckle noise in synthetic

Manuscript received June 6, 1989; revised February 8, 1991. This paper was recommended by Associate Editor K. H. Tzou.

The authors are with the Department of Electrical Engineering, Colorado State University, Fort Collins, CO 80523.

IEEE Log Number 9101497.

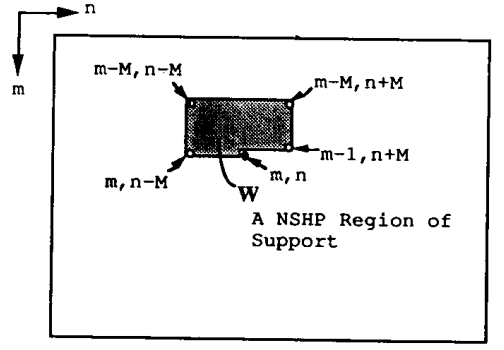

Fig. 1. The NSHP ROS

aperture radar (SAR) imagery. Other important work in this area is in [7]-[9].

In this paper, a 2-D recursive parameter identification process is derived using the 2-D extension of the stochastic Newton approach [10] which can be used to estimate the parameters of an AR model with NSHP region of support (ROS). In Section II, the 2-D image and degradation models with NSHP regions of support are arranged in a state-space form, in which the state propagates in two dimensions. For this dynamical model the space-varying 2-D Kalman filtering equations are given in Section III, which take into account the nonstationarity in the image field. The development of the recursive parameter identification process using the stochastic Newton approach is also presented in Section III. In Section IV, the implementation of the adaptive Kalman filtering on a real world image is considered, and the simulation results are provided.

\section{State-Space Modeling of Image and Degradation Processes}

Consider an image of size $\mathrm{N} \times \mathrm{N}$ which is scanned row by row from left-to-right and top-to-bottom. The image is assumed to be represented by a zero-mean Markovian field and modeled by an $M \times M$-order AR process with NSHP ROS given by:

$$
u(m, n)=\sum_{p, q \in W} a_{p, q} u(m-p, n-q)+e(m, n)
$$

where $u(m, n)$ represents the pixel intensity at location $(m, n)$; $e(m, n)$ is a white noise sequence that drives the process; $a_{p, q}$ 's are the reflection coefficients of the AR model; and the prediction window is $W=\{(p, q): 1<p \leqslant M,-M \leqslant q \leqslant M$ and $p=$ $0,1 \leqslant q \leqslant M\}$. This region is shown in Fig. 1 . The statistics of the driving process $e(m, n)$ are given as:

$$
\begin{gathered}
E[e(m, n)]=0 \\
E[e(m+k, n+l) e(m, n)]=\sigma_{e}^{2} \delta(k, l)
\end{gathered}
$$

where $\sigma_{e}^{2}$ is the variance of the error $e(m, n) ; \delta(k, l)$ is the Kronecker delta function and $E$ is the expectation operator. The orthogonality principle gives

$$
E[e(m, n) u(m-p, n-q)]=0 \quad \forall(p, q) \in W .
$$

The current "local state" vector of size $\left(2 M^{2}+2 M+1\right) \times 1$ consists of all the pixels in the ROS, $W$, of the model. This is 
defined by

$$
\begin{aligned}
X(m, n)=[ & u(m, n) u(m, n-1) \\
& \cdots u(m, n-M) u(m-1, n+M) \cdots \\
& \cdots u(m-1, n-M) \cdots u(m-M, n+M) \\
& \cdots u(m-M, n-M)]^{t} .
\end{aligned}
$$

This local state vector can be related to the past local state vectors by the following local 2-D state-space model [11], i.e.,

$$
X(m, n)=A^{1,0} X(m, n-1)+A^{0,1} X(m-1, n+M)+B e(m, n)
$$

where

$$
\begin{aligned}
A^{1,0} & \equiv\left[\begin{array}{ccccc}
A_{0}^{\prime} & A_{1}^{\prime} & A_{2}^{\prime} & \cdots & A_{M}^{\prime} \\
0 & I^{\prime} & 0 & \cdots & 0 \\
0 & \cdot & \cdot & \cdots & \cdot \\
\cdot & \cdot & \cdot & \cdots & \cdot \\
0 & \cdot & \cdot & \cdots & I^{\prime}
\end{array}\right] \\
A^{0,1} & =\left[\begin{array}{ccccc}
A_{1}^{\prime \prime} & A_{2}^{\prime \prime} & \cdots & A_{M}^{\prime \prime} & 0 \\
I_{1}^{\prime \prime} & 0 & \cdots & \cdot & 0 \\
\cdot & \cdot & \cdots & \cdot & \cdot \\
\cdot & \cdot & \cdots & \cdot & \cdot \\
\cdot & \cdot & \cdots & I^{\prime \prime} & 0
\end{array}\right] \\
B & \equiv\left[\begin{array}{llllll}
1 & 0 & \cdots & 0 & \cdots & 0
\end{array}\right]^{t} .
\end{aligned}
$$

The elemental block of $A^{1,0}$ and $A^{0,1}$ matrices are given by

$$
\begin{aligned}
& A_{0}^{\prime}=\left[\begin{array}{ccccc}
a_{01} & a_{02} & \cdots & a_{0 M} & 0 \\
1 & 0 & \cdots & \vdots & 0 \\
\cdot & \cdot & \cdots & : & \cdot \\
\cdot & \cdot & \cdots & 1 & 0
\end{array}\right]_{(M+1)(M+1)} \\
& A_{i}^{\prime}=\left[\begin{array}{cccccc}
a_{i-(M-1)} & \cdots & a_{i 0} & \cdots & a_{i M} & 0 \\
0 & \cdots & 0 & \cdots & \cdot & 0 \\
\cdot & \cdots & \cdot & \cdots & \cdot & \cdot \\
0 & \cdots & \cdot & \cdots & \cdot & 0
\end{array}\right]_{(M+1)(2 M+1)} \\
& i=1, \cdots M \quad(7 \mathrm{~b}) \\
& I^{\prime} \equiv\left[\begin{array}{cccc}
0 & . & \cdots & 0 \\
1 & 0 & \cdots & 0 \\
0 & 1 & \cdots & 0 \\
. & \cdot & \cdots & . \\
0 & \cdot & 1 & 0
\end{array}\right] \\
& A_{1}^{\prime \prime}=\left[\begin{array}{cccc}
a_{1-M} & 0 & \cdots & 0 \\
0 & 0 & \cdots & 0 \\
\cdot & \cdot & \cdots & \cdot \\
0 & \cdot & \cdots & 0
\end{array}\right]_{(M+1)(M+1)} \\
& A_{i}^{\prime \prime}=\left[\begin{array}{ccccccc}
0 & \cdots & 0 & a_{2,-M} & 0 & \cdots & 0 \\
0 & \cdots & 0 & 0 & 0 & \cdots & 0 \\
. & \cdots & . & \cdot & \cdot & \cdots & \cdot \\
0 & \cdots & \cdot & \cdot & \cdot & \cdots & 0
\end{array}\right]_{(M+1)(2 M+1)} \\
& i=2, \cdots M \\
& I_{1}^{\prime \prime}=\left[\begin{array}{llll}
1 & 0 & \cdots & 0 \\
0 & 0 & \cdots & 0 \\
\cdot & \cdot & \cdots & 0 \\
0 & \cdot & \cdots & 0
\end{array}\right]_{(2 M+1)(M+1)} \\
& I^{\prime \prime}=\left[\begin{array}{ll}
0 & I_{1}^{\prime \prime}
\end{array}\right]_{(2 M+1)(2 M+1)}
\end{aligned}
$$

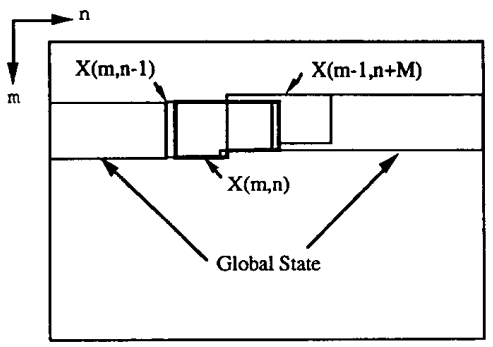

Fig. 2. Local and global states in the NSHP state-space model.

The past local state vectors in this model, i.e., vectors $X(m, n-1)$ and $X(m-1, n+M)$ are needed for the computation of $X(m, n)$ as shown in Fig. 2. The "global state" vector at stage $(m, n)$ on the other hand, has to include all the necessary $(N)$ vectors $X(m-1, k), k \in[n+M, N-1]$ and $X(m, l), l \in$ $[0, n+M-1]$.

The degradation process consisting of LSI blur and additive WG noise can be modeled as

$$
y(m, n)=\sum_{p, q \in W} h(p, q) u(m-p, n-q)+v(m, n)
$$

where $y(m, n), h(m, n)$, and $v(m, n)$ are, respectively, the corrupted image, the point spread function (PSF) of the blur with NSHP support confined in $W$, and the additive WG noise with zero mean and variance $\sigma_{v}^{2}$. This equation can be written in matrix vector form as

$$
y(m, n)=C X(m, n)+v(m, n)
$$

where

$$
\begin{aligned}
C=[h(0,0) h(0,1) \cdots h(0, M) h(1,-M) \\
\\
\cdots h(1, M) \cdots h(M,-M) \cdots h(M, M)] .
\end{aligned}
$$

Thus equations (5) and (10) would give the complete 2-D dynamic model for the process.

\section{Space-Varying 2-D Kalman Filter and Parameter Identification}

For simplicity of derivations let us map the 2-D array $(m, n)$ to a 1-D array using the index mapping of $k=m+(n-1) N$, $\forall m, n \in[1, N]$. Using this mapping we have

$$
\begin{aligned}
X(m, n) & \rightarrow X(k) \\
X(m, n-1) & \rightarrow X(k-1) \\
X(m-1, n+M) & \rightarrow X(k-N+M) .
\end{aligned}
$$

This applies to any other vector in the model. Thus the 2-D space-varying dynamic model for the process becomes

$$
\begin{aligned}
X(k) & =A^{1,0}(\phi) X(k-1)+A^{0,1}(\phi) X(k-N+M)+B e(k) \\
y(k) & =C X(k)+v(k)
\end{aligned}
$$

where $\phi$ represents the unknown parameter vector obtained from the lexicographical arrangement of the reflection coefficients $a_{i j}$ 's. The Kalman filter equations for this model are then

\section{(טגו}

Publicity Chairman: Dr. Hari r Rada

\section{- High definition television}

- Image processing
- Solid stote circuits

- VLSI design and applications. 
given [6] by

$$
\begin{aligned}
\hat{X}(k)= & A^{1,0}(\phi) \hat{\hat{X}}(k-1)+A^{0,1}(\phi) \hat{\hat{X}}(k-N+M) \\
P_{b}(k):= & E\left[(X(k)-\hat{X}(k))(X(k)-\hat{X}(k))^{t}\right] \\
= & A^{1,0}(\phi) P_{a}(k-1) A^{1,0^{t}}(\phi)+A^{0,1}(\phi) \\
& \cdot P_{a}(k-N+M) A^{0,1^{t}}(\phi)+B B^{t} \sigma_{e}^{2} \\
K(k, \phi)= & P_{b}(k) C^{t}\left[C P_{b}(k) C^{t}+\sigma_{v}^{2} I\right]^{-1} \\
\hat{X}(k)= & \hat{X}(k)+K(k, \phi)[y(k)-C \hat{X}(k)] \\
P_{a}(k):= & E\left[(X(k)-\hat{\hat{X}}(k))(X(k)-\hat{\hat{X}}(k))^{t}\right] \\
= & {[I-K(k) C] P_{b}(k) }
\end{aligned}
$$

where $P_{b}(k)$ and $P_{a}(k)$ are, respectively, the $a$ priori and the $a$ posteriori error covariance matrices; $K(k, \phi)$ is the Kalman gain matrix; and $\hat{X}$ and $\hat{X}$ denote the $a$ priori and the a posterior $i$ estimates of the local state vectors. To account for the effects of nonstationarity in the image field the parameters of the dynamic model (12) have to be identified locally at each processing window during the filtering. This calls for an efficient recursive process for on-line estimation of these parameters. Now let us rewrite the updating equation (13d) into the "filtered form," i.e.,

$$
\begin{aligned}
\hat{X}(k) & =\hat{X}(k)+K(k, \phi)[y(k)-C \hat{X}(k)] \\
& =[I-K(k, \phi) C] \hat{X}(k)+K(k, \phi) y(k) \\
y(k \mid \hat{\phi}) & =\hat{y}(k)=C \hat{X}(k) .
\end{aligned}
$$

Given this model the natural way to measure the goodness of the estimates is the prediction error $z(k, \phi)=y(k)-y(k \mid \hat{\phi})$. A reasonable figure of merit for measuring the size of this vector is the quadratic norm

$$
N(\phi)=E\left[\frac{1}{2} z^{t}(k, \phi) \Pi^{\prime} z(k, \phi)\right]
$$

where $\Pi$ is a positive definite matrix. The optimal choice of $\Pi$ is the covariance matrix of the true prediction error $z(k, \phi)$ denoted by $R_{z}(k, \phi)$. The above quadratic criterion can be recursively minimized for $\phi$ by using stochastic Newton method [10]. This leads to the following recursive equation

$$
\phi(k)=\phi(k-1)+v(k) H^{-1}(k) \Delta(k, \phi) R_{z}^{-1}(k, \phi) z(k, \phi)
$$

where $H(k)$ is the Hessian (second derivative) of $N(\phi)$ wrt $\phi$ and can be computed recursively [10] using

$H(k)=H(k-1)+v(k)$

$$
\cdot\left[\Delta(k, \phi) R_{z}^{-1}(k, \phi) \Delta^{t}(k, \phi)-H(k-1)\right] .
$$

In this equations $\{v(k)\}$ is the gain sequence that should satisfy $v(k)>0, \quad \lim _{M_{0} \rightarrow \infty} \sum_{k=0}^{M_{0}} v(k) \rightarrow \infty, \quad \lim _{M_{0} \rightarrow \infty} \sum_{k=0}^{M_{0}} v^{2}(k)<\infty$

where $M_{0}=N^{2}$ is a large number representing the total number of pixels in the image. The third condition ensures that the effects of noisy measurements are eliminated asymptotically; and the second condition guarantees the existence of a target. The choices for the gain sequence $\{v(k)\}$ and the initial value of
TABLE I

The 2-D Recursive Parameter Identification Algortthm

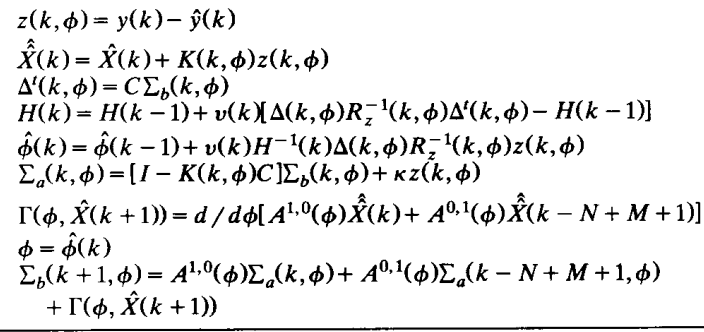

$H(k)$ are discussed in [10]. The gradient of the prediction is defined by

$$
\Delta(k, \phi)=\left[\frac{d}{d \phi} \hat{y}(k)\right]^{t}
$$

using (14b) we get

$$
\Delta^{\prime}(k, \phi)=C \sum_{b}(k, \phi)
$$

where

$$
\sum_{b}(k, \phi):=\frac{d}{d \phi} \hat{X}(k)
$$

Matrix $\sum_{b}$ can be computed recursively using

$\sum_{b}(k, \phi)=A^{1,0}(\phi) \sum_{a}(k-1, \phi)+A^{0,1}(\phi)$

$$
\cdot \sum_{a}(k-N+M, \phi)+\Gamma(\phi, \hat{X}(k))
$$

where

$$
\begin{aligned}
\Gamma(\phi, \hat{X}(k)) & :=\frac{d}{d \phi}\left[A^{1.0}(\phi) \hat{\hat{X}}(k-1)\right. \\
& \left.+A^{0,1}(\phi) \hat{\hat{X}}(k-N+M)\right]_{\phi=\hat{\phi}(k) .}
\end{aligned}
$$

A recursive equation for matrix $\Sigma_{a}$ can be obtained by taking derivative of (14a), i.e.,

$$
\sum_{a}(k, \phi):=\frac{d}{d \phi} \hat{\hat{X}}(k)=[I-K(k, \phi) C] \sum_{b}(k, \phi)+\kappa z(k, \phi)
$$

where

$$
\kappa:=\frac{d}{d \phi} K(k, \phi) .
$$

The gradient matrix $\kappa$ can either be obtained [5], [10] recursively or by using backward difference approximation. The complete recursive identification algorithm is given in order in Table I.

Choice of the Gain Sequence: The gain sequence, $\{v(k)\}$, in the adaptation equation has a significant influence on both the transient behavior of the algorithm and the accuracy of the estimation. Let us define a new sequence, $\{\lambda(k)\}$, which is called "forgetting factor" $[10]$ as

$$
\lambda(k)=\frac{v(k-1)[1-v(k)]}{v(k)}
$$




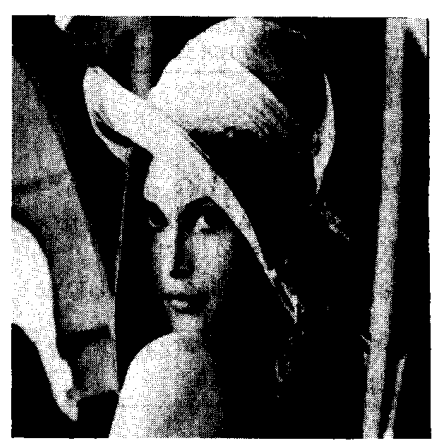

Fig. 3. Original "girl" image.

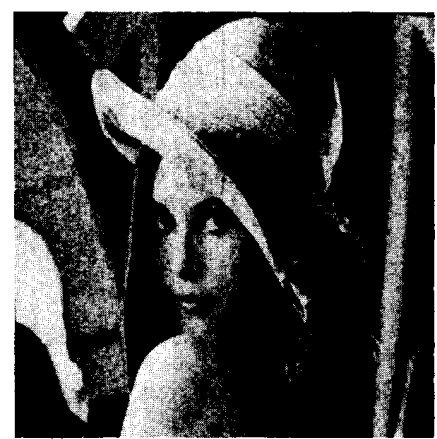

Fig. 4. Corrupted girl image $(\mathrm{SNR}=5 \mathrm{~dB})$.

or

$$
v(k)=\frac{1}{1+\lambda(k) / v(k-1)}
$$

which grows exponentially to 1 . It is suggested [10] that for most practical situations where the order of the AR model is not high, the following numerical method be used to find $\{\lambda(k)\}$ :

$$
\lambda(k)=0.99 \lambda(k-1)+0.01, \quad \lambda(0)=0.95 .
$$

For a large order models, $\lambda(k)$ should grow more slowly to 1 . The choice of the initial estimate of $H(k)$ is also discussed in great details in [10]. If no prior information about the variance of the output innovation and covariance of $\phi(0)$ is available the reasonable choice for $H(0)$ is found to be

$$
H(0)=\rho^{-1} I \text {. }
$$

Small values of $\rho$ result in slower convergence. This corresponds to the case when too much confidence is given to the initial estimates $\phi(0)$.

\section{Implementation and Results}

In this section the proposed 2-D adaptive Kalman filtering algorithm is used to remove the effects of additive WG noise from an image. The test image chosen is the "girl" image in Fig. 3 , which is degraded by adding a zero-mean WG noise to obtain SNR of $5 \mathrm{~dB}$. The resultant image is shown in Fig. 4. Initial estimates of the AR model parameters are obtained globally from the noisy image. In addition, we set $\hat{X}(0)=0, P_{a}(0)=\sigma_{u}^{2} I$,

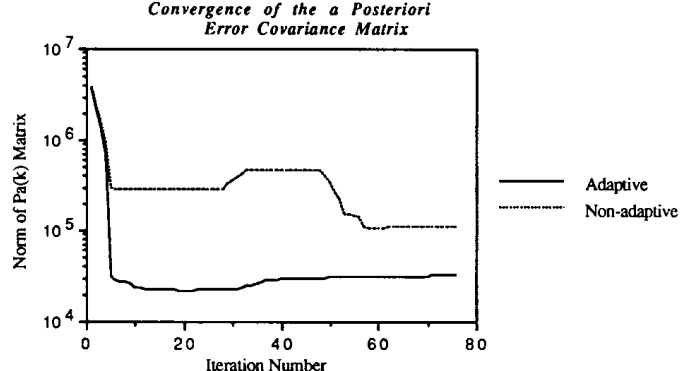

Fig. 5. Accuracy in the estimation for adaptive and nonadaptive Kalman filters.

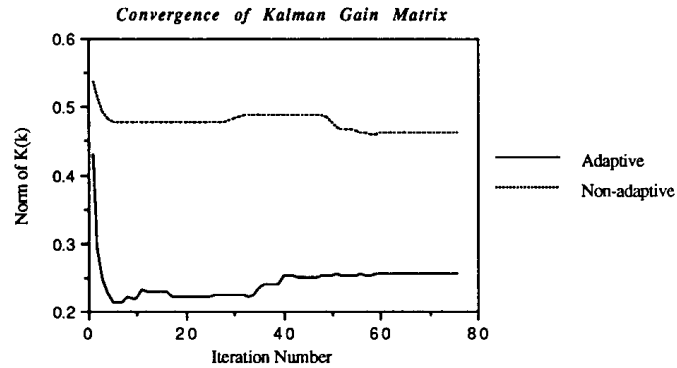

Fig. 6. Convergence and tracking behavior of adaptive and nonadaptive Kalman filters.

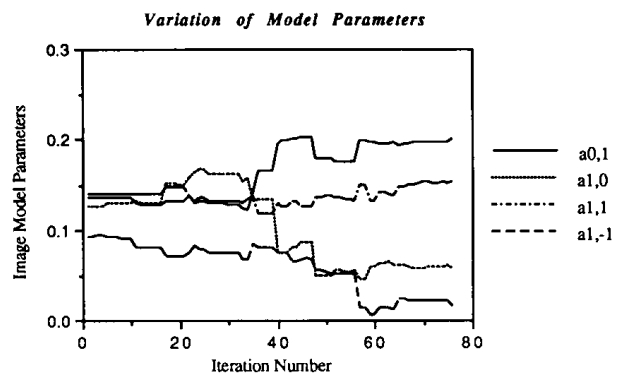

Fig. 7. Parameter variation in the adaptive Kalman filter.

$H(0)=I$ and the initial values of $\sum_{b}(0)$ and $\Gamma(\phi(0), \hat{X}(0))$ as

$$
\sum_{b}(0)=\Gamma(\phi(0), \hat{X}(0))=\left[\begin{array}{cccc}
-\mu_{u} & \mu_{u} & \cdots & \mu_{u} \\
0 & 0 & \cdots & 0 \\
0 & 0 & \cdots & 0
\end{array}\right] .
$$

The adaptation process is restarted at the beginning of each new row. A comparison is made between the Euclidean norms of $P_{a}(k)$ and $K(k)$ matrices for both the nonadaptive and adaptive cases. The norm of $P_{a}(k)$ matrix, which indicates the accuracy in estimation is plotted for the first 75 pixels of row 256 of the image for both cases as shown in Fig. 5. The plot of norm of $K(k)$, which shows convergence behavior and tracking characteristic of the process, is shown in Fig. 6. These plots clearly indicate the accuracy in estimation and the fast convergence of our method when compared with the conventional non-adaptive Kalman filters. The plots of variations of four significant model parameters, i.e., $a_{0,1}, a_{1,0}, a_{1,1}$, and $a_{1,-1}$, are also shown in Fig.

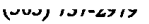

Publicity Chairman: Dr. Hari C RadA.

\section{- High definition television}

- Image processing
- Solid stote circuits

- VLSI design and applications. 


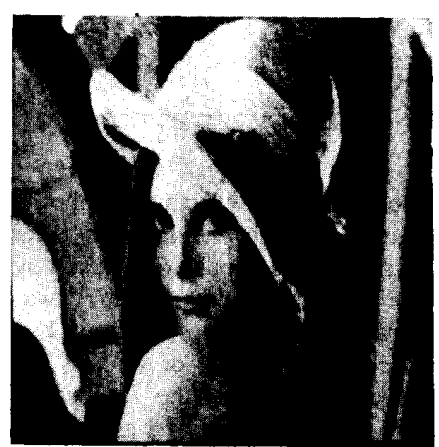

Fig. 8. Processed image using adaptive Kalman filter $(\mathrm{SNR}=11 \mathrm{~dB})$.

7. These plots demonstrate the edge-dependent characteristic of the adaptation algorithm. The resultant filtered image is shown in Fig. 8. The SNR for this image is measured to be $11 \mathrm{~dB}$, which indicates 6-dB improvement.

\section{ConClusion}

This paper presents a 2-D space-varying Kalman filter for NSHP image models. The parameter identification is accomplished using the stochastic Newton approach which accounts for the non-stationarity in the image field and adjusts the model parameters on-line based upon the local spatial activities within each processing window. Simulation results on an image are provided, which show the improvements in the performance comparing with those of the nonadaptive methods.

\section{REFERENCES}

[1] H. Kaufman et al., "Estimation and identification of two-dimensional images," IEEE Trans. Automat. Contr., vol. AC-28, pp. 745-756, July 1983 .

[2] A. M. Tekalp et al., "Fast recursive estimation of parameters of a space-varying autoregressive image model," IEEE Trans. Acoust., Speech, Signal Processing, vol. ASSP-33, pp. 469-472, Apr. 1985.

[3] S. A. Rajala and R. J. P. De Figueiredo, "Adaptive nonlinear image restoration by a modified Kalman filtering approach," IEEE Trans. Acoust., Speech, Signal Processing, vol. ASSP-29, pp. 1033-1042, Oct. 1981.

[4] A. M. Tekalp, H. Kaufman, and J. W. Woods, "Edge-Adaptive Kalman filtering for image restoration with ringing suppression," IEEE Trans. Acoust., Speech, Signal Processing, vol. ASSP-37, pp. 892-899, June 1989.

[5] M. R. Azimi-Sadjadi, "Two-dimensional adaptive block recursive filtering," in Proc. Int. Symp. Circuits and Systems, pp. 446-450, May 1987.

[6] M. R. Azimi-Sadjadi and S. Bannour, "Two-dimensional adaptive block Kalman filtering of SAR imagery," submitted to IEEE Trans. Geosci. Remote Sensing.

[7] J. Biemond, F. G. Van Der Putten, and J. W. Woods, "Identification and restoration of images with symmetric noncausal blurs," IEEE Trans. Circuits Syst., vol. CAS-35, pp. 385-393, Apr. 1988.

[8] D. Angwin and H. Kaufman, "Image restoration by using reduced order models," in special issues of EURASIP, 1988

[9] R. L. Lagendijk, A. K. Katsagellos, and J. Biemond, "Constrained iterative identification and restoration of images," Proc. EUSIPCO-88, pp. 1585-1588, 1988.

[10] L. Ljung and T. Soderstrom, Theory and Practice of Recursive Identification. Cambridge, MA: MIT Press, 1983.
[11] S. Y. Kung et al., "New results in 2-D systems theory, Part II: 2-D state-space models-realization and the notions of controllability, observability, and minimality," Proc. IEEE, vol. 65, pp. 945-961, June 1977.

\section{Realization of Voltage-Controlled Impedances}

Raj Senani and D. R. Bhaskar

\begin{abstract}
Some time back, Nay and Budak presented a technique employing JFET and op-amps for obtaining voltage-controlled positive/ negative resistance exhibiting wide dynamic range and low distortion. In this paper, two new op-amp-JFET configurations are proposed, which not only realize voltage-controlled resistances but, unlike the Nay and Budak configuration, make it possible to realize voltage-controlled inductance and voltage-controlled capacitance elements, too. Experimental results are given to demonstrate the practical validity of the theoretical formulations.
\end{abstract}

\section{INTRODUCTION}

In [1] and [2], Nay and Budak (NB) presented a technique of obtaining linear positive/negative voltage-controlled resistance (VCR) having wide dynamic range and low distortion, with linear range considerably greater than other known FET VCR's. The proposition of [1] and [2] is reproduced here in Fig. 1. The input resistance of the circuit, when FET is confined to operate in non-saturated region, is given by

$$
R_{\text {in }}=\frac{v_{D}}{i_{D}}=\frac{V_{p}^{2}}{I_{D S S}\left(V_{c}-2 V_{p}\right) a}
$$

so that when $a>0, R_{\text {in }}$ presents a voltage-controlled positive resistance (VCPR) [1], whereas with $a<0$, voltage-controlled negative resistance (VCNR) is realized [2]. Note that extension of linear range has been made possible because whereas usual FET VCR provides linear VCR for only small $v_{D S}$ (typically $\leqslant 100 \mathrm{mV}$ ), the VCR of Fig. 1 can handle voltage swing of the order of several volts (see fig. 6 of [1]) while the FET inside the VCR is still confined to handle only a small fraction $a v_{D}(a \ll 1)$ of the input voltage $v_{D}$.

In the following, alternative configurations are presented that not only provide VCR's but can also provide voltage-controlled inductance and voltage-controlled capacitance (by appropriate choice of two circuit impedances) which are not realizable from the NB circuits [1], [2] in any way.

\section{Realization of Voltage-Controlled Positive IMPEDANCES}

\section{A. The Proposed Configurations}

The first proposition is shown in Fig. 2(a). Although we employ an extra op-amp and five more resistors than NB circuit

Manuscript received June 28, 1989; revised April 22, 1991. This paper was recommended by Associate Editor T. K. Ishii.

$R$. Senani is with the Department of Electronics and Communication Engineering, Delhi Institute of Technology, Delhi 110006, India.

D. R. Bhaskar is with the Electrical Engineering Department, Delhi College of Engineering, Delhi 110006, India.

IEEE Log Number 9101498. 